Marcin Przystalski (Słupia Wielka)

\title{
HYPOTHESIS TESTING IN UNBALANCED TWO-FOLD NESTED RANDOM MODELS
}

Abstract. In many applications of linear random models to multilevel data, it is of interest to test whether the random effects variance components are zero. In this paper we propose approximate tests for testing significance of variance components in the unbalanced two-fold nested random model in the presence of non-normality. In the derivations of the asymptotic distributions of the test statistics, as an intermediate result, the explicit form of the asymptotic covariance matrix of the vector of mean squares in this model is obtained. We also study the influence of some special type of designs on the asymptotic covariance matrix and on the distribution of the proposed test statistics.

1. Introduction. In many medical, agricultural and biological studies multilevel data are collected. Multilevel data arise when there is hierarchical or clustered structure of the data. In [16], an experiment was described in which the relative effectiveness of two drugs with respect to some specified criterion was studied. The experiment was designed in such a way that drug 1 was to be administered to $n$ patients from each of hospitals $1,2,3$, while drug 2 was to be administered to $n$ patients from hospitals 4, 5, 6. In this example, the hospital effects were nested under the drug factor. One can observe that, in the above example, each drug was tested in the same number of hospitals and in each hospital the drug was administered to the same number of patients, thus the experiment can be described by a balanced two-fold nested model. However, if in this example one assumes that drug 1 was administered to $n_{11}$ patients from hospital 1 and $n_{12}$ patients from

2010 Mathematics Subject Classification: Primary 62F03.

Key words and phrases: asymptotic covariance matrix, asymptotic normality, hypothesis testing.

Received 31 July 2015; revised 30 November 2015.

Published online 8 February 2016. 
hospital 2, while drug 2 was administered to $n_{24}, n_{25}$ and $n_{26}$ patients from hospitals 4,5 and 6, respectively, then to describe the experiment one should use an unbalanced two-fold nested model. In general, if we denote by $y_{i j k}$ the $k$ th observation $\left(k=1, \ldots, n_{i j}\right)$ for the $i$ th level of the nesting factor $(i=1, \ldots, v)$ measured in the $j$ th level of the nested factor $\left(j=1, \ldots, b_{i}\right)$, then an unbalanced two-fold nested model can be written as

$$
y_{i j k}=\mu+\alpha_{i}+\beta_{i j}+e_{i j k},
$$

where $\mu$ denotes the general mean, $\alpha_{i}$ is the nesting effect, $\beta_{i j}$ is the nested effect, and $e_{i j k}$ is a random error. In this paper, both $\alpha_{i}$ and $\beta_{i j}$ are assumed to be random. Usually, it is assumed that $\alpha_{i} \sim N\left(0, \sigma_{a}^{2}\right), \beta_{i j} \sim N\left(0, \sigma_{b}^{2}\right)$ and $e_{i j k} \sim N\left(0, \sigma_{e}^{2}\right)$. However, in practice the normality assumption is often violated. For this reason, in this paper we will assume that $\alpha_{i}, \beta_{i j}$ and $e_{i j k}$ are independent random variables with zero mean and variances $\sigma_{a}^{2}, \sigma_{b}^{2}$ and $\sigma_{e}^{2}$, respectively.

For inference on variance components, many testing procedures have been proposed in the literature (see e.g. [6, 10, 13]). In recent years, many tests concerning the significance of variance components in balanced and unbalanced linear random models in the presence of non-normality were constructed by using joint distribution of the mean squares. In [1, for balanced data, Arkitras and Arnold showed that the vector of mean squares in a one-way model and a two-way model converges to a multivariate normal distribution with mean vector zero and an appropriate covariance matrix. Using this result they obtained the asymptotic distribution for the $F$-test in those two models. A similar result was obtained by Güven [8] for an unbalanced two-way random model without interaction in the presence of non-normality. Using the same approach, Güven [7] constructed an asymptotic $F$-test in a one-fold nested model. For an unbalanced two-fold nested model, in the normal case, some test procedures for inference on variance components have been presented in [10; however, for non-normal unbalanced two-fold nested models no such test procedures have been proposed.

For this reason, in this paper we propose approximate tests for the hypotheses $H_{0}: \sigma_{b}^{2}=0$ and $H_{0}: \sigma_{a}^{2}=0$ in model 1.1 . Using the Main Theorem in [14] and the delta method we obtain the asymptotic distributions of the test statistics. Additionally, as an intermediate result, we obtain the explicit form of the asymptotic covariance matrix of the vector of mean squares in model (1.1). Furthermore, we will study the influence of some special type of designs on the asymptotic covariance matrix and the distribution of the test statistics.

This paper is organized as follows. In Section 2 we introduce some notation which will be used throughout the paper. Section 3 presents the model, assumptions and some auxilary results. In Section 4 the main results are 
established. In Section 5, we study the influence of some special type of designs on the asymptotic covariance matrix and asymptotic distribution of the $F$-statistics. Simulation studies are given in Section 6 . In Section 7 we provide a discussion. The proofs are postponed until Section 8 .

2. Notation. In this section we fix the notation which will be used in the subsequent sections. Throughout the paper, we will denote matrices and vectors by bold letters $\mathbf{A}$ and $\boldsymbol{a}$. The notations $\mathbf{J}_{n}$ and $\mathbf{1}_{n}$ stand respectively for the $n \times n$ matrix and $n \times 1$ vector of ones. $\bigoplus$ is the symbol of direct sum of matrices, i.e. for given square matrices $\mathbf{A}_{i}(i=1, \ldots, m), \bigoplus_{i=1}^{m} \mathbf{A}_{i}=$ $\operatorname{diag}\left(\mathbf{A}_{1}, \ldots, \mathbf{A}_{m}\right)$.

$E(\cdot), \operatorname{Cov}(\cdot, \cdot)$ mean expected value and covariance, respectively. Depending on the context, $\operatorname{Var}(\cdot)$ will denote either the variance of a random variable or the covariance matrix of a random vector.

Let $X$ be a zero-mean random variable. Then $k_{x}$ will denote the kurtosis parameter for the distribution of $X$, defined by $k_{x}=E\left(X^{4}\right) / \sigma^{4}-3$.

Let $\left\{X_{n}\right\}$ be a sequence of random variables. Then

$$
X_{n}=\mathrm{o}_{p}(1) \Leftrightarrow X_{n} \stackrel{p}{\rightarrow} 0, \quad \text { as } n \rightarrow \infty .
$$

\section{Model and assumptions}

3.1. Statistical model. Let us define $N, n_{i}$. and $b$. as

$$
N=\sum_{i=1}^{v} \sum_{j=1}^{b_{i}} n_{i j}, \quad n_{i} .=\sum_{j=1}^{b_{i}} n_{i j}, \quad b .=\sum_{i=1}^{v} b_{i} .
$$

Then in the matrix notation of [10], model (1.1) can be expressed as

$$
\boldsymbol{y}=\mu \mathbf{1}_{N}+\mathbf{Z}_{1} \boldsymbol{\alpha}+\mathbf{Z}_{2} \boldsymbol{\beta}+\boldsymbol{e},
$$

where $\boldsymbol{y}$ is a vector of observations, $\mu$ denotes the general mean, $\boldsymbol{\alpha}$ and $\boldsymbol{\beta}$ are vectors of random effects, and $\boldsymbol{e}$ denotes a vector of random errors. We assume that the random vectors $\boldsymbol{\alpha}, \boldsymbol{\beta}$ and $\boldsymbol{e}$ are independent. The matrices $\mathbf{Z}_{1}(N \times v)$ and $\mathbf{Z}_{2}(N \times b$. $)$ are design matrices for $\boldsymbol{\alpha}$ and $\boldsymbol{\beta}$, respectively, and are equal to

$$
\begin{aligned}
& \mathbf{Z}_{1}=\operatorname{diag}\left(\mathbf{1}_{n_{1}}, \ldots, \mathbf{1}_{n_{v} .}\right) \\
& \mathbf{Z}_{2}=\operatorname{diag}\left(\mathbf{1}_{n_{11}}, \ldots, \mathbf{1}_{n_{1 b_{1}}}, \mathbf{1}_{n_{21}}, \ldots, \mathbf{1}_{n_{2 b_{2}}}, \ldots, \mathbf{1}_{n_{v 1}}, \ldots \mathbf{1}_{n_{v b_{v}}}\right) .
\end{aligned}
$$

Without loss of generality one may assume that $n_{i j} \geq 1$ for all $i$ and $j$, since if $n_{i j}=0$ then the corresponding level of nested effect does not occur in the experiment and hence does not have to be taken into account.

Often in model (3.1) the following hypotheses are of interest: $H_{01}: \sigma_{b}^{2}=0$ and $H_{02}: \sigma_{a}^{2}=0$. Alternatively, they can be written in the following way:

$$
H_{01}^{\prime}: \rho_{2}=0 \quad \text { and } H_{02}^{\prime}: \rho_{1}=0 \text {, }
$$

where $\rho_{1}=\sigma_{a}^{2} / \sigma_{e}^{2}$ and $\rho_{2}=\sigma_{b}^{2} / \sigma_{e}^{2}$. 
Usually to test $H_{01}$ and $H_{02}$ the test statistics $F_{1}=M S B / M S E$ and $F_{2}=M S A / M S B$ respectively are used, where $M S A=S S A /(v-1), M S B=$ $S S B /(b .-v)$ and $M S E=S S E /(N-b$. $)$. The sums of squares $S S A, S S B$ and $S S E$ are

$$
S S A=\boldsymbol{y}^{\prime} \mathbf{P}_{1} \boldsymbol{y}, \quad S S B=\boldsymbol{y}^{\prime} \mathbf{P}_{2} \boldsymbol{y}, \quad S S E=\boldsymbol{y}^{\prime} \mathbf{P}_{3} \boldsymbol{y},
$$

where $\mathbf{P}_{i}(i=1,2,3)$ are orthogonal projection matrices and can be written as (see [10])

$$
\begin{aligned}
& \mathbf{P}_{1}=\bigoplus_{i=1}^{v} \frac{1}{n_{i} .} \mathbf{J}_{n_{i} .}-\frac{1}{N} \mathbf{J}_{N}, \quad \mathbf{P}_{2}=\bigoplus_{i=1}^{v}\left[\bigoplus_{j=1}^{b_{i}} \frac{1}{n_{i j}} \mathbf{J}_{n_{i j}}-\frac{1}{n_{i} .} \mathbf{J}_{n_{i}}\right], \\
& \mathbf{P}_{3}=\mathbf{I}_{N}-\bigoplus_{i=1}^{v} \bigoplus_{j=1}^{b_{i}} \frac{1}{n_{i j}} \mathbf{J}_{n_{i j}} .
\end{aligned}
$$

3.2. Assumptions and some useful results. In this subsection we give some assumptions and some general results needed in the subsequent sections.

The following assumptions will be needed throughout the paper.

(A1) For every $\varepsilon>0$, we have $E\left|\alpha_{i}\right|^{4+\varepsilon}<\infty, E\left|\beta_{i j}\right|^{4+\varepsilon}<\infty$ and $E\left|e_{i j k}\right|^{4+\varepsilon}<\infty\left(i=1, \ldots, v ; j=1, \ldots, b_{i} ; k=1, \ldots, n_{i j}\right)$.

(A2) The number $v$ of treatments tends to infinity in such a way that the $n_{i j}$ 's take values from a finite set of distinct positive integers.

(A3) Define $l_{v}(t)$ and $m_{v}(t)$ to be the number of occurrences of $m_{t}$ in $\left(n_{1}, \ldots, n_{v}\right)$ and of $r_{l}$ in $\left(\sum_{j}^{b_{1}} n_{1 j}^{-1}, \ldots, \sum_{j}^{b_{v}} n_{v j}^{-1}\right)$, respectively. Then $l_{v}(t) / v \rightarrow p_{t}$ and $m_{v}(t) / v \rightarrow q_{t}$.

(A4) There exists $M>1$ such that $1 \leq b_{i} \leq M$ for each $1 \leq i \leq v$.

REMARK 3.1. As pointed out in [8], assumption (A2) implies that both $n_{i}$. and $\sum_{j}^{b_{i}} n_{i j}^{-1}(i=1, \ldots, v)$ take values from finite discrete sets of positive numbers. Hence, one may assume that $n_{i}, i=1, \ldots, v$, take values from a set

$$
S_{1}=\left\{m_{1}, \ldots, m_{l}\right\}
$$

and $\sum_{j=1}^{b_{i}} n_{i j}^{-1}, i=1, \ldots, v$, take values from

$$
S_{2}=\left\{r_{1}, \ldots, r_{l}\right\} \text {. }
$$

In the proofs of the main results the following lemmas will be needed. The first two are modifications of Lemmas 3.1 an 3.2 in [8] to the setting of this paper, and can be proved using similar arguments. 
Lemma 3.2. Let

$$
\psi_{k}=\lim _{v \rightarrow \infty} \frac{1}{v} \sum_{i=1}^{v} n_{i .}^{k}, \quad k=\mp 1, \mp 2, \quad \omega=\lim _{v \rightarrow \infty} \frac{1}{v} \sum_{i=1}^{v} \sum_{j=1}^{b_{i}} \frac{1}{n_{i j}} .
$$

Then under assumptions (A2)-(A4),

$$
\psi_{k}=\sum_{t=1}^{l} m_{t}^{k} p_{t} \quad \text { and } \quad \omega=\sum_{t=1}^{l} r_{t}^{k} q_{t}
$$

where $l$ is the number of different elements of the sets $S_{1}$ and $S_{2}$ defined in (3.5) and (3.6).

LEMma 3.3. The number $v$ of levels of the nesting factor and the number $N$ of observations are of the same order, and the limits

$$
\begin{aligned}
\zeta & =\lim _{N \rightarrow \infty} \frac{v}{N}, \quad \gamma_{k}=\lim _{N \rightarrow \infty} \frac{1}{N} \sum_{i=1}^{v} n_{i}^{k} . \quad \text { for } k=\mp 1, \mp 2, \\
\eta & =\lim _{N \rightarrow \infty} \frac{1}{N} \sum_{i=1}^{v} \sum_{j=1}^{b_{i}} \frac{1}{n_{i j}}
\end{aligned}
$$

are positive and finite.

LEMMA 3.4. Under assumptions (A2)-(A4) the limits

$$
\begin{aligned}
\varphi & =\lim _{N \rightarrow \infty} \frac{b .}{N}, \quad \delta_{k 2}=\lim _{N \rightarrow \infty} \frac{1}{N} \sum_{i=1}^{v} \sum_{j=1}^{b_{i}} \frac{n_{i j}^{2}}{n_{i .}^{k}} \quad \text { for } k=0,1,2, \\
\delta_{l, l+2} & =\lim _{N \rightarrow \infty} \frac{1}{N} \sum_{i=1}^{v} \sum_{j=1}^{b_{i}} \frac{n_{i j}^{l+2}}{n_{i}^{l} .} \quad \text { for } l=1,2, \\
\vartheta & =\lim _{N \rightarrow \infty} \frac{1}{N} \sum_{i=1}^{v} \frac{1}{n_{i .}^{2}}\left(\sum_{j=1}^{b_{i}} n_{i j}^{2}\right)^{2}, \quad \delta=\lim _{N \rightarrow \infty} \frac{1}{N} \sum_{i=1}^{v} \frac{b_{i} .}{n_{i} .}
\end{aligned}
$$

are finite and positive.

REMARK 3.5. In practice the limits in Lemmas 3.23 .4 are unknown. Thus, they should be replaced by their finite sample counterparts.

4. Main results. To derive the asymptotic distributions of the test statistics $F_{1}$ and $F_{2}$ (defined in Section 3.1) we will use the delta method. We first find the asymptotic covariance and the joint distribution of $\sqrt{N}[M S A, M S B, M S E]^{\prime}$.

Let $\boldsymbol{\theta}=[M S A, M S B, M S E]^{\prime}$. 
THEOREM 4.1. Under assumptions (A1)-(A4), the asymptotic covariance matrix of $\sqrt{N} \boldsymbol{\theta}$ is equal to

where

$$
\boldsymbol{\Sigma}=\sigma_{e}^{4}\left(\mathbf{\Psi}_{1}+k_{a} \rho_{1}^{2} \mathbf{\Psi}_{2}+k_{b} \rho_{2}^{2} \mathbf{\Psi}_{3}+k_{e} \mathbf{\Psi}_{4}\right),
$$

$\mathbf{\Psi}_{1}=$

$$
\begin{aligned}
& {\left[\begin{array}{lll}
\frac{2\left(\rho_{1}^{2} \gamma_{2}+2 \rho_{1} \rho_{2} \delta_{02}+2 \rho_{1}+2 \rho_{2} \delta_{12}+\rho_{2}^{2} \vartheta+\zeta\right)}{\zeta^{2}} & \frac{2 \rho_{2}^{2}\left(\delta_{13}-\vartheta\right)}{\zeta(\varphi-\zeta)} & 0 \\
\frac{2 \rho_{2}^{2}\left(\delta_{13}-\vartheta\right)}{\zeta(\varphi-\zeta)} & \frac{2\left(\rho_{2}^{2}\left(\delta_{02}-2 \delta_{13}+\vartheta\right)+2 \rho_{2}\left(1-\delta_{12}\right)+\varphi-\zeta\right)}{(\varphi-\zeta)^{2}} & 0 \\
0 & 0 & \frac{2}{1-\varphi}
\end{array}\right], } \\
& \Psi_{2}=\left[\begin{array}{lll}
\frac{\gamma_{2}}{\zeta^{2}} & 0 & 0 \\
0 & 0 & 0 \\
0 & 0 & 0
\end{array}\right], \mathbf{\Psi}_{3}=\left[\begin{array}{lll}
\frac{\delta_{24}}{\zeta^{2}} & \frac{\delta_{13}-\delta_{24}}{\zeta(\varphi-\zeta)} & 0 \\
\frac{\delta_{13}-\delta_{24}}{\zeta(\varphi-\zeta)} & \frac{\delta_{02}-2 \delta_{13}+\delta_{24}}{(\varphi-\zeta)^{2}} & 0 \\
0 & 0 & 0
\end{array}\right]
\end{aligned}
$$

and

$$
\Psi_{4}=\left[\begin{array}{lll}
\frac{\gamma-1}{\zeta^{2}} & \frac{\delta-\gamma-1}{\zeta(\varphi-\zeta)} & \frac{\varphi-\delta}{\zeta(1-\varphi)} \\
\frac{\delta-\gamma-1}{\zeta(\varphi-\zeta)} & \frac{\eta+\gamma-1-2 \delta}{(\varphi-\zeta)^{2}} & \frac{\varphi-\eta-\zeta+\delta}{(1-\varphi)(\varphi-\zeta)} \\
\frac{\varphi-\delta}{\zeta(1-\varphi)} & \frac{\varphi-\eta-\zeta+\delta}{(1-\varphi)(\varphi-\zeta)} & \frac{1-2 \varphi+\eta}{(1-\varphi)^{2}}
\end{array}\right]
$$

Proposition 4.2. Under assumptions (A1)-(A4),

$$
\sqrt{N}(\boldsymbol{\theta}-\boldsymbol{\Gamma}) \stackrel{d}{\rightarrow} N(\mathbf{0}, \boldsymbol{\Sigma}),
$$

where $\boldsymbol{\Gamma}=\left[\sigma_{e}^{2}\left(\rho_{1} \zeta^{-1}+\rho_{2} \delta_{12} \zeta^{-1}+1\right), \sigma_{e}^{2}\left(1+\rho_{2}\left(1-\delta_{12}\right)(\varphi-\zeta)^{-1}\right), \sigma_{e}^{2}\right]^{\prime}$.

Combining Proposition 4.2 with the delta method (see e.g. [12]) we get the following:

TheOREM 4.3. Suppose that assumptions (A1)-(A4) hold.

(i) We have

as $N \rightarrow \infty$, where

$$
\sqrt{N}\left(F_{1}-\left(1+\frac{\rho_{2}\left(1-\delta_{12}\right)}{\varphi-\zeta}\right)\right) \stackrel{d}{\rightarrow} N\left(0, \sigma_{H_{1}}^{2}\right),
$$

$$
\begin{aligned}
\sigma_{H_{1}}^{2}= & \frac{2\left(\rho_{2}^{2}\left(\delta_{02}-2 \delta_{13}+\vartheta\right)+2 \rho_{2}\left(1-\delta_{12}\right)+\varphi-\zeta\right)}{(\varphi-\zeta)^{2}} \\
& +\frac{2}{1-\varphi}\left(1+\frac{\rho_{2}\left(1-\delta_{12}\right)}{\varphi-\zeta}\right)^{2}+k_{b} \rho_{2}^{2} \frac{\delta_{02}-2 \delta_{13}+\delta_{24}}{(\varphi-\zeta)^{2}} \\
& +k_{e}\left[\frac{\eta+\gamma_{-1}-2 \delta}{(\varphi-\zeta)^{2}}-\frac{2\left(1+\frac{\rho_{2}\left(1-\delta_{12}\right)}{\varphi-\zeta}\right)(\varphi-\eta-\zeta+\delta)}{(1-\varphi)(\varphi-\zeta)}\right. \\
& \left.+\frac{\left(1+\frac{\rho_{2}\left(1-\delta_{12}\right)}{\varphi-\zeta}\right)^{2}(1-2 \varphi+\eta)}{(1-\varphi)^{2}}\right] .
\end{aligned}
$$


(ii) We have

$$
\begin{aligned}
& \sqrt{N}\left(F_{2}-\left(1+\frac{\rho_{1} \zeta^{-1}+\rho_{2} \delta_{12} \zeta^{-1}-\rho_{2}\left(1-\delta_{12}\right)(\varphi-\zeta)^{-1}}{1+\rho_{2}\left(1-\delta_{12}\right)(\varphi-\zeta)^{-1}}\right)\right) \\
& \text { as } N \rightarrow \infty \text {, where }
\end{aligned}
$$

$$
\begin{aligned}
\sigma_{H_{2}}^{2} & =a\left[\frac{2\left(\rho_{1}^{2} \gamma_{2}+2 \rho_{1} \rho_{2} \delta_{02}+\rho_{2}^{2} \vartheta+\zeta\right)}{\zeta^{2}}-\frac{4 d \rho_{2}^{2}\left(\delta_{13}-\vartheta\right)}{c \zeta}\right. \\
& +\frac{2 d^{2}\left(\rho_{2}^{2}\left(\delta_{02}-2 \delta_{13}+\vartheta\right)+2\left(1-\delta_{12}\right)+(\varphi-\zeta)\right)}{c^{2}} \\
& +k_{a} \rho_{1}^{2} \frac{\gamma_{2}}{\zeta^{2}}+k_{b} \rho_{2}^{2}\left(\frac{\delta_{24}}{\zeta^{2}}-\frac{2 d\left(\delta_{13}-\delta_{24}\right)}{c \zeta}+\frac{d^{2}\left(\delta_{02}-2 \delta_{13}+\delta_{24}\right)}{c^{2}}\right) \\
& \left.+k_{e}\left(\frac{\gamma_{-1}}{\zeta^{2}}-\frac{2 d\left(\delta-\gamma_{-1}\right)}{c \zeta}+\frac{d^{2}\left(\eta+\gamma_{-1}-2 \delta\right)}{c^{2}}\right)\right]
\end{aligned}
$$

and $c=\varphi-\zeta+\rho_{2}\left(1-\delta_{12}\right), a=(\varphi-\zeta)^{2} c^{-2}, d=1+\rho_{1} \zeta^{-1}+\delta_{12} \rho_{2} \zeta^{-1}$.

Corollary 4.4. Assume that the assumptions of Theorem 4.3 are satisfied.

(i) Under $H_{01}: \rho_{2}=0$,

where

$$
\sqrt{N}\left(F_{1}-1\right) \stackrel{d}{\rightarrow} N\left(0, \sigma_{H_{01}}^{2}\right),
$$

$$
\begin{aligned}
\sigma_{H_{01}}^{2}= & \frac{2}{\varphi-\zeta}+\frac{2}{1-\varphi} \\
& +k_{e}\left(\frac{\eta+\gamma_{-1}-2 \delta}{(\varphi-\zeta)^{2}}-\frac{2(\varphi-\eta-\zeta+\delta)}{(1-\varphi)(\varphi-\zeta)}+\frac{1-2 \varphi+\eta}{(1-\varphi)^{2}}\right) .
\end{aligned}
$$

(ii) Under $H_{02}: \rho_{1}=0$,

$$
\sqrt{N}\left(F_{2}-\left(1+\frac{\rho_{2} \delta_{12} \zeta^{-1}-\rho_{2}\left(1-\delta_{12}\right)(\varphi-\zeta)^{-1}}{1+\rho_{2}\left(1-\delta_{12}\right)(\varphi-\zeta)^{-1}}\right)\right) \stackrel{d}{\rightarrow} N\left(0, \sigma_{H_{02}}^{2}\right),
$$

where

$$
\begin{aligned}
\sigma_{H_{02}}^{2}=a\left[\frac{2\left(\rho_{2}^{2} \vartheta+\zeta\right)}{\zeta^{2}}-\frac{4 d \rho_{2}^{2}\left(\delta_{13}-\vartheta\right)}{c \zeta}\right. \\
+\frac{2 d^{2}\left(\rho_{2}^{2}\left(\delta_{02}-2 \delta_{13}+\vartheta\right)+2\left(1-\delta_{12}\right)+(\varphi-\zeta)\right)}{c^{2}} \\
+k_{b} \rho_{2}^{2}\left(\frac{\delta_{24}}{\zeta^{2}}-\frac{2 d\left(\delta_{13}-\delta_{24}\right)}{c \zeta}+\frac{d^{2}\left(\delta_{02}-2 \delta_{13}+\delta_{24}\right)}{c^{2}}\right) \\
\left.+k_{e}\left(\frac{\gamma_{-1}}{\zeta^{2}}-\frac{2 d\left(\delta-\gamma_{-1}\right)}{c \zeta}+\frac{d^{2}\left(\eta+\gamma_{-1}-2 \delta\right)}{c^{2}}\right)\right],
\end{aligned}
$$

$a$ and $c$ are defined in Theorem 4.3, and $d=1+\rho_{2} \delta_{12} \zeta^{-1}$. 
5. Special case. As we have seen in Section 4, the correlations between the mean squares in the unbalanced two-fold nested random model depend mainly on kurtoses, and most of the off-diagonal elements of $\boldsymbol{\Psi}_{1}$ are zero. However, when one looks at the correlation between $M S A$ and $M S B$ one can observe that $\Psi_{1,12} \neq 0$. A similar observation has been made by Cummings and Gaylor [4] (see also [10, Example 3.5.1]) for unbalanced linear mixed models under normality.

However in the normal case, for some designs the mean squares $M S A$ and $M S B$ are uncorrelated. Cummings and Gaylor [4 described several designs and studied their impact on the correlation between $M S A$ and $M S B$ as well as on the distribution of the sum of squares. In one of the designs they assumed that for a given $i(i=1, \ldots, v)$ and $j \neq j^{\prime}$ one has $n_{i j}=n_{i j^{\prime}}$. In [10], this type of design is called a partially balanced design $(P B D)$.

In the same example, Cummings and Gaylor pointed out that for a partially balanced design, $M S A$ and $M S B$ are independent under normality. In our case, for a PBD, from Lemma 3.4 it follows that $\delta_{13}=\vartheta$ and the asymptotic covariance matrix $\boldsymbol{\Sigma}$ given Theorem 4.1 can be written as:

Corollary 5.1. Suppose that the assumptions of Theorem 4.1 hold. Additionally, let $n_{i j}=n_{i j^{\prime}}$ for $i=1, \ldots, v$ and $j, j^{\prime}=1, \ldots, b_{i}, j \neq j^{\prime}$. Then the asymptotic covariance matrix of $\sqrt{N} \boldsymbol{\theta}$ is equal to

$$
\boldsymbol{\Sigma}=\sigma_{e}^{4}\left(\widetilde{\mathbf{\Psi}}_{1}+k_{a} \rho_{1}^{2} \widetilde{\mathbf{\Psi}}_{2}+k_{b} \rho_{2}^{2} \widetilde{\mathbf{\Psi}}_{3}+k_{e} \widetilde{\mathbf{\Psi}}_{4}\right)
$$

where

$$
\begin{aligned}
& \widetilde{\Psi}_{1}= \\
& {\left[\begin{array}{lll}
\frac{2\left(\rho_{1}^{2} \gamma_{2}+2 \rho_{1} \rho_{2} \delta_{02}+2 \rho_{1}+2 \rho_{2} \delta_{12}+\rho_{2}^{2} \vartheta+\zeta\right)}{\zeta^{2}} & 0 & 0 \\
0 & \frac{2\left(\rho_{2}^{2}\left(\delta_{02}-\delta_{13}\right)+2 \rho_{2}\left(1-\delta_{12}\right)+\varphi-\zeta\right)}{(\varphi-\zeta)^{2}} & 0 \\
0 & 0 & \frac{2}{1-\varphi}
\end{array}\right],}
\end{aligned}
$$

and $\widetilde{\boldsymbol{\Psi}}_{2}, \widetilde{\boldsymbol{\Psi}}_{3}$ and $\widetilde{\boldsymbol{\Psi}}_{4}$ are as in Theorem 4.1 .

If, in Theorem 4.3, we assume that the experiment was organized as a $\mathrm{PBD}$, then we get the following:

Corollary 5.2. Suppose that the assumptions of Theorem 4.1 hold. Additionally, let $n_{i j}=n_{i j^{\prime}}$ for $i=1, \ldots, v$ and $j, j^{\prime}=1, \ldots, b_{i}, j \neq j^{\prime}$.

(i) We have

$$
\sqrt{N}\left(F_{1}-\left(1+\frac{\rho_{2}\left(1-\delta_{12}\right)}{\varphi-\zeta}\right)\right) \stackrel{d}{\rightarrow} N\left(0, \sigma_{H_{1}}^{2}\right)
$$


as $N \rightarrow \infty$, where

$$
\begin{aligned}
\sigma_{H_{1}}^{2}= & \frac{2\left(\rho_{2}^{2}\left(\delta_{02}-\delta_{13}\right)+2 \rho_{2}\left(1-\delta_{12}\right)+\varphi-\zeta\right)}{(\varphi-\zeta)^{2}} \\
& +\frac{2}{1-\varphi}\left(1+\frac{\rho_{2}\left(1-\delta_{12}\right)}{\varphi-\zeta}\right)^{2}+k_{b} \rho_{2}^{2} \frac{\delta_{02}-2 \delta_{13}+\delta_{24}}{(\varphi-\zeta)^{2}} \\
& +k_{e}\left[\frac{\eta+\gamma_{-1}-2 \delta}{(\varphi-\zeta)^{2}}-\frac{2\left(1+\frac{\rho_{2}\left(1-\delta_{12}\right)}{\varphi-\zeta}\right)(\varphi-\eta-\zeta+\delta)}{(1-\varphi)(\varphi-\zeta)}\right. \\
& \left.+\frac{\left(1+\frac{\rho_{2}\left(1-\delta_{12}\right)}{\varphi-\zeta}\right)^{2}(1-2 \varphi+\eta)}{(1-\varphi)^{2}}\right] .
\end{aligned}
$$

(ii) We have

$$
\begin{aligned}
\sqrt{N} & \left(F_{2}-\left(1+\frac{\rho_{1} \zeta^{-1}+\rho_{2} \delta_{12} \zeta^{-1}-\rho_{2}\left(1-\delta_{12}\right)(\varphi-\zeta)^{-1}}{1+\rho_{2}\left(1-\delta_{12}\right)(\varphi-\zeta)^{-1}}\right)\right) \\
\text { as } N & \rightarrow \infty, \text { where } \\
\sigma_{H_{2}}^{2} & =a\left[\frac{2\left(\rho_{1}^{2} \gamma_{2}+2 \rho_{1} \rho_{2} \delta_{02}+\rho_{2}^{2} \vartheta+\zeta\right)}{\zeta^{2}}\right) \\
& +\frac{2 d^{2}\left(\rho_{2}^{2}\left(\delta_{02}-\delta_{13}\right)+2\left(1-\delta_{12}\right)+(\varphi-\zeta)\right)}{c^{2}} \\
& +k_{a} \rho_{1}^{2} \frac{\gamma_{2}}{\zeta^{2}}+k_{b} \rho_{2}^{2}\left(\frac{\delta_{24}}{\zeta^{2}}-\frac{2 d\left(\delta_{13}-\delta_{24}\right)}{c \zeta}+\frac{d^{2}\left(\delta_{02}-2 \delta_{13}+\delta_{24}\right)}{c^{2}}\right) \\
& \left.+k_{e}\left(\frac{\gamma_{-1}}{\zeta^{2}}-\frac{2 d\left(\delta-\gamma_{-1}\right)}{c \zeta}+\frac{d^{2}\left(\eta+\gamma_{-1}-2 \delta\right)}{c^{2}}\right)\right]
\end{aligned}
$$

and $a, c, d$ are defined in Theorem 4.3 .

6. Simulation studies. In this section we will study the performance of the approximate tests described in Section 5.

In the simulation study we will consider a design similar to the one in [10, Example 3.5.1, Design 3]. Suppose the number $n_{i j}$ of observations corresponding to the $j$ th level of the nested factor within the $i$ th level of the nesting factor has the following values: $n_{11}=n_{21}=n_{31}=n_{41}=1, n_{22}=8$, $n_{32}=10, n_{42}=5, n_{43}=6, n_{44}=7$. Thus, $N=40, v=4, b_{1}=1$, $b_{2}=b_{3}=2$ and $b_{4}=4$. In the simulations, we consider four combinations of distributions for the random effects and errors:

(i) Normal-Normal-Normal (N-N-N),

(ii) N-N-CE, where CE represents centralized exponential distribution, i.e. $X-1$, where $X \sim \operatorname{Exp}(1)$, 
(iii) CE-CE-CE,

(iv) SN-SN-SN, where SN represents the skew-normal distribution (for the definition see [2]) with the following parameters:

I $[1]_{4}^{\prime}$ for $\boldsymbol{\alpha}$, where $[1]_{4}^{\prime}=[1,1,1,1]^{\prime},[1]_{9}^{\prime}$ for $\boldsymbol{\beta}$ and $[1]_{40}^{\prime}$ for $\boldsymbol{e}$;

II $[-1,1 / 2,1 / 3,1]^{\prime},[-1,-1 / 2,0,1,1,1,1,1,1]^{\prime}$ and $[1]_{40}^{\prime}$;

III $[1 / 2,-1 / 2,1 / 2,-1 / 2]$, $[1]_{9}^{\prime}$ and $[0]_{40}^{\prime}$.

In all cases as a scale matrix $\mathbf{K}$ we took the identity matrix of the appropriate order, i.e. $\mathbf{K}=\mathbf{I}_{l}, l=4,9,40$.

The kurtoses for the distributions used in the simulations are given in Table 1, where the kurtoses for skew-normal distributions were calculated from the formulas in [2, Section 4].

Table 1. Kurtoses of the distributions

\begin{tabular}{lcccccc}
\hline Kurtosis & N-N-N & N-N-CE & CE-CE-CE & \multicolumn{3}{c}{ SN-SN-SN } \\
\cline { 5 - 7 } & & & & I & II & III \\
\hline$k_{a}$ & 0 & 0 & 6 & 0.3050 & 0.1853 & 0.0617 \\
$k_{b}$ & 0 & 0 & 6 & 0.5098 & 0.4290 & 0.5098 \\
$k_{e}$ & 0 & 6 & 6 & 0.7609 & 0.7609 & 0 \\
\hline
\end{tabular}

First, we test the hypothesis $H_{0}: \sigma_{b}^{2}=0$, or equivalently $H_{0}: \rho_{2}=0$. In the simulations we assumed that the true values in model (3.1) are $\mu=$ $\sigma_{a}^{2}=\sigma_{e}^{2}=1$.

Based on 10000 simulations, the estimated size of test $P\left(F_{1}>u_{\alpha} \mid \rho_{2}=0\right)$ was calculated as the number of times $F_{1}$ exceeds the cut-off point $u_{\alpha}$ divided by 10000 , where $u_{\alpha}$ was calculated using Corollary 4.4(i) and it is given by

$$
u_{\alpha}=\frac{\sigma_{01}}{\sqrt{N}} z_{\alpha}+1,
$$

and $z_{\alpha}$ is the upper $1-\alpha$ quantile of the standard normal distribution.

The estimated sizes of the test for the nominal levels $\alpha=0.01,0.05,0.1$ are reported in Table 2.

Table 2. Estimated size

\begin{tabular}{lcccccc}
\hline \multirow{2}{*}{$\begin{array}{l}\text { Nominal } \\
\text { level }\end{array}$} & N-N-N & N-N-CE & CE-CE-CE & \multicolumn{3}{c}{ SN-SN-SN } \\
\cline { 5 - 7 } & & & & I & II & III \\
\hline$\alpha=0.01$ & 0.0466 & 0.0402 & 0.0427 & 0.0373 & 0.0342 & 0.0451 \\
$\alpha=0.05$ & 0.0837 & 0.0614 & 0.0649 & 0.0764 & 0.0736 & 0.0896 \\
$\alpha=0.1$ & 0.1267 & 0.0913 & 0.0927 & 0.1095 & 0.1131 & 0.1256 \\
\hline
\end{tabular}

Next, we look at the approximate powers for the following alternatives $\rho_{2}=0.1,0.25,0.5,0.75,1,2,4$ for two nominal levels $\alpha=0.01,0.05$. The 
approximate powers were calculated using Theorem 4.3(i) as

$$
P\left(F_{1}>u_{\alpha} \mid \rho_{2}>0\right) \cong 1-\Phi\left(\frac{u_{\alpha}-\left(1+\frac{\rho_{2}\left(1-\delta_{12}\right)}{\varphi-\zeta}\right)}{\sigma_{1}}\right)
$$

and are reported in Tables 3 and 4 .

Table 3. Approximate power (nominal level 0.01)

\begin{tabular}{llllccc}
\hline & N-N-N & N-N-CE & CE-CE-CE & \multicolumn{3}{c}{ SN-SN-SN } \\
\cline { 5 - 7 } & & & & I & II & III \\
\hline$\rho_{2}=0.1$ & 0.0470 & 0.0441 & 0.0468 & 0.0730 & 0.0762 & 0.0885 \\
$\rho_{2}=0.25$ & 0.2821 & 0.1362 & 0.1676 & 0.2489 & 0.2491 & 0.2811 \\
$\rho_{2}=0.5$ & 0.5189 & 0.3291 & 0.3568 & 0.4785 & 0.4771 & 0.5182 \\
$\rho_{2}=0.75$ & 0.6390 & 0.4675 & 0.4758 & 0.6036 & 0.6045 & 0.6321 \\
$\rho_{2}=1$ & 0.7105 & 0.5580 & 0.5427 & 0.6734 & 0.6759 & 0.6995 \\
$\rho_{2}=2$ & 0.8080 & 0.7186 & 0.6531 & 0.7794 & 0.7813 & 0.7929 \\
$\rho_{2}=4$ & 0.8536 & 0.7967 & 0.7094 & 0.8300 & 0.8321 & 0.8378 \\
\hline
\end{tabular}

Table 4. Approximate power (nominal level 0.05)

\begin{tabular}{lcccccc}
\hline & \multirow{2}{*}{ N-N-N } & N-N-CE & CE-CE-CE & \multicolumn{3}{c}{ SN-SN-SN } \\
\cline { 5 - 7 } & & & & I & II & III \\
\hline$\rho_{2}=0.1$ & 0.1962 & 0.1240 & 0.1302 & 0.1776 & 0.1788 & 0.1972 \\
$\rho_{2}=0.25$ & 0.4149 & 0.2639 & 0.2870 & 0.3810 & 0.3818 & 0.4201 \\
$\rho_{2}=0.5$ & 0.6113 & 0.4506 & 0.4609 & 0.5791 & 0.5785 & 0.6084 \\
$\rho_{2}=0.75$ & 0.7038 & 0.5660 & 0.5522 & 0.6730 & 0.6747 & 0.6954 \\
$\rho_{2}=1$ & 0.7541 & 0.6478 & 0.6036 & 0.7241 & 0.7266 & 0.7412 \\
$\rho_{2}=2$ & 0.8276 & 0.7557 & 0.6833 & 0.8026 & 0.8044 & 0.8130 \\
$\rho_{2}=4$ & 0.8628 & 0.8143 & 0.7237 & 0.8405 & 0.8427 & 0.8473 \\
\hline
\end{tabular}

Now, for the same design and combinations of distributions we will study the performance of the approximation test for $H_{0}: \rho_{1}=0$. We assumed that the true values of parameters in model (3.1) were $\mu=\sigma_{e}^{2}=1$ and $\sigma_{b}^{2}=1,4$. Once again, based on 10000 simulations we calculated the estimated size of the test, but now the cut-off point $u_{\alpha}$ calculated using Corollary 4.4(ii) and was equal to

$$
u_{\alpha}=\frac{\sigma_{02}}{\sqrt{N}} z_{\alpha}+\left(1+\frac{\rho_{2} \delta_{12} \zeta^{-1}-\rho_{2}\left(1-\delta_{12}\right)(\varphi-\zeta)^{-1}}{1+\rho_{2}\left(1-\delta_{12}\right)(\varphi-\zeta)^{-1}}\right),
$$

where $z_{\alpha}$ is the upper $1-\alpha$ quantile of the standard normal distribution.

For the same nominal levels the estimated sizes of the test are reported in Table 5 . 
Table 5. Estimated size

\begin{tabular}{lcccccc}
\hline \multirow{2}{*}{$\begin{array}{l}\text { Nominal } \\
\text { level }\end{array}$} & \multirow{2}{*}{ N-N-N } & N-N-CE & CE-CE-CE & \multicolumn{4}{c}{ SN-SN-SN } \\
\cline { 5 - 7 } & & & & I & II & III \\
\hline & & & $\rho_{2}=1$ \\
$\alpha=0.01$ & 0.1327 & 0.1273 & 0.0948 & 0.1123 & 0.1115 & 0.1135 \\
$\alpha=0.05$ & 0.1790 & 0.1815 & 0.1371 & 0.1538 & 0.1541 & 0.1599 \\
$\alpha=0.1$ & 0.2227 & 0.2162 & 0.1629 & 0.1905 & 0.1927 & 0.1925 \\
& & & $\rho_{2}=4$ & & & \\
$\alpha=0.01$ & 0.1114 & 0.1046 & 0.0842 & 0.1008 & 0.1050 & 0.1015 \\
$\alpha=0.05$ & 0.1529 & 0.1534 & 0.1213 & 0.1439 & 0.1445 & 0.1442 \\
$\alpha=0.1$ & 0.1862 & 0.1884 & 0.1497 & 0.1788 & 0.1795 & 0.1815 \\
\hline
\end{tabular}

Because the estimated sizes of this test were rather far from the nominal levels, we do not give the results on the power of the test.

7. Discussion. In this paper we derived the explicit form of the asymptotic covariance matrix of the vector of mean squares in a non-normal unbalanced two-fold nested random model, and we constructed approximate procedures for testing the hypotheses $H_{0}: \sigma_{b}^{2}=0$ and $H_{0}: \sigma_{a}^{2}=0$. Furthermore, we studied the influence of some special type of designs on the asymptotic covariance matrix and on the distributions of the test statistics.

All results were obtained under the assumption that the number of levels of nesting effects goes to infinity. In the proofs of the main results, besides the standard assumptions, we only required the convergence of some partial sums of sample sizes. We used similar assumptions to the ones used in [8], which are equivalent to assumptions (i)-(iii) in [14]. In the proofs of the main results we did not require assumptions (iv) and (v) of [14], because they follow from assumptions (A2)-(A4).

Using the covariance matrix of the mean squares, we constructed approximate procedures for testing the hypotheses $H_{0}: \sigma_{a}^{2}=0$ and $H_{0}: \sigma_{b}^{2}=0$ in an unbalanced two-fold nested model in the presence of non-normality. We showed that the test statistics are asymptotically normal. One can see that the asymptotic variances of the test statistics (see Theorem 4.3 and Corollary 4.4 as well the covariance matrix of the vector of mean squares (Theorem 4.1) depend on the kurtoses $k_{a}, k_{b}$ and $k_{e}$, which in some cases could be known from some previous studies. However, in general, these parameters are unknown. To overcome this problem, one may use a similar approach to that proposed in [9]. We plan to explore this problem in a future work.

To check the performance of the proposed test procedures we carried out simulation studies. As mentioned earlier, all results were developed when the 
sample size $N$ goes to infinity. In practice, one applies the asymptotic results when $N \geq 30$ (see [5, p. 58]). In our simulations, all calculations were carried out for $N=40$.

In the simulation studies the approximate test for testing $H_{0}: \sigma_{b}^{2}=0$ was performing well. For all combinations the estimated sizes of the test were close to the nominal levels. The best results in terms of estimated size were obtained when all random effects and errors follow an exponential distribution. The results obtained for SN-SN-SN I and II also had the estimated size close to the nominal levels, but in comparison to CE-CE-CE had higher approximate power for both nominal levels studied. N-N-N had slightly bigger values of approximate power for both nominal levels than the skew-normal combinations. This can be partly explained by the fact that for SN-SN-SN both I and II had kurtoses close to one (see Table 1).

The results for testing $H_{0}: \sigma_{a}^{2}=0$ were not as satisfactory as those for $H_{0}: \sigma_{b}^{2}=0$. The best results, in terms of the estimated size, were obtained for CE-CE-CE. This can be partly explained by high values of kurtoses of random effects. Based on the simulation studies, one can observe that for bigger values of the nuisance parameter $\rho_{2}$ the estimated sizes of the test are closer to the nominal levels. This can be explained by the fact that the distribution of the test statistics depends on the nuisance parameter. To eliminate the influence of this parameter one could use the concept of generalized $p$-value (see e.g. [10], [13]). A different method of solving this problem would be to construct other test statistics. We plan to explore this problem.

Because most of the experiments are organized according to some design, we also studied the influence of PBD designs on the asymptotic covariance matrix $\boldsymbol{\Sigma}$ and on the distribution of the test statistics. It was pointed out that under the PBD assumption, the matrix $\boldsymbol{\Psi}_{1}$ is diagonal, which is in agreement with the results obtained in [10, Example 3.5.1].

Finally, it should be noted that testing significance of variance components in random two-fold nested models is only one of several hypothesis testing problems for which further statistical research is needed. For example, the problem of testing the null hypothesis $H_{0}: \sigma_{a}^{2}=\sigma_{b}^{2}$ against the alternative $H_{1}: \sigma_{a}^{2}>\sigma_{b}^{2}$ should be investigated. Such a test would be very interesting for practitioners, e.g. for researchers working in agronomy.

8. Proofs. In this section we give the proofs of the statements formulated in Sections 3 and 4.

Proof of Lemma 3.4. We have

$$
v \leq b .=\sum_{i=1}^{v} b_{i} \leq M \sum_{i=1}^{v} 1=M v \quad(\text { by }(\mathrm{A} 3)) .
$$


Thus, by Lemma 3.3 it follows that

$$
\lim _{N \rightarrow \infty} \frac{b}{N} \leq M \zeta<\infty
$$

Since $\zeta>0$, one obtains

$$
\lim _{N \rightarrow \infty} \frac{b}{N} \geq \zeta>0 .
$$

Now, from $1 \leq n_{i j} \leq n_{i}$. and (A3), for $k=0,1,2$ one has

$$
\begin{aligned}
\frac{1}{N} \sum_{i=1}^{v} \frac{1}{n_{i}^{k}} \leq & \frac{1}{N} \sum_{i=1}^{v} \sum_{j=1}^{b_{i}} \frac{n_{i j}^{2}}{n_{i}^{k}} \leq \frac{1}{N} \sum_{i=1}^{v} \sum_{j=1}^{b_{i}} \frac{n_{i .}^{2}}{n_{i}^{k}} \\
& = \begin{cases}\frac{1}{N} \sum_{i=1}^{v} b_{i} \cdot n_{i}^{2} \leq \frac{M}{N} \sum_{i=1}^{v} n_{i \cdot}^{2}, & k=0, \\
\frac{1}{N} \sum_{i=1}^{v} b_{i} \cdot n_{i} \leq \frac{M}{N} \sum_{i=1}^{v} n_{i} ., & k=1, \\
\frac{b .}{N}, & k=2 .\end{cases}
\end{aligned}
$$

Thus, finiteness of $\delta_{02}$ follows from $\gamma_{2}, \delta_{12}<\infty$ since $\gamma_{1}<\infty$, whereas finiteness of $\delta_{22}$ follows from $\varphi<\infty$. The positivity of $\delta_{k 2}, k=1,2$, follows from $\gamma_{k}>0, k=1,2$ (see Lemma 3.3), while positivity of $\delta_{02}$ follows from $\zeta>0$.

Using similar arguments one can show that $\delta$ and $\delta_{l, l+2}$ are positive finite.

Finally, using the fact that $1 \leq \sum_{j=1}^{b_{i}} n_{i j}^{2} \leq n_{i}^{2}$ and assumption (A3) one obtains

$$
\frac{1}{N} \sum_{i=1}^{v} n_{i}^{2} \leq \frac{1}{N} \sum_{i=1}^{v} \frac{\left(\sum_{j=1}^{b_{i}} n_{i j}^{2}\right)^{2}}{n_{i}^{2} .} \leq \frac{M}{N} \sum_{i=1}^{v} n_{i}^{2} .
$$

Hence, by Lemma 3.3 it follows that $\vartheta$ is positive and finite.

Proof of Theorem 4.1. Denote

$$
\boldsymbol{\theta}=[M S A, M S B, M S E]^{\prime}, \quad \boldsymbol{\Theta}=[S S A, S S B, S S E]^{\prime} .
$$

Then,

$$
\sqrt{N} \boldsymbol{\theta}=\boldsymbol{\Omega}_{n} N^{-1 / 2} \boldsymbol{\Theta}
$$

and by basic properties of the covariance matrix (see e.g. [3])

$$
\operatorname{Var}(\sqrt{N} \boldsymbol{\theta})=\boldsymbol{\Omega}_{N} \frac{1}{N} \operatorname{Var}(\boldsymbol{\Theta}) \boldsymbol{\Omega}_{N},
$$

where

$$
\boldsymbol{\Omega}_{N}=\left[\begin{array}{ccc}
\frac{N}{v-1} & 0 & 0 \\
0 & \frac{N}{b .-v} & 0 \\
0 & 0 & \frac{N}{N-b .}
\end{array}\right]
$$


By Lemmas 3.3 and 3.4 ,

$$
\lim _{N \rightarrow \infty} \boldsymbol{\Omega}_{N}=\left[\begin{array}{ccc}
\frac{1}{\zeta} & 0 & 0 \\
0 & \frac{1}{\varphi-\zeta} & 0 \\
0 & 0 & \frac{1}{1-\varphi}
\end{array}\right]=\boldsymbol{\Omega} .
$$

Hence, to prove the theorem one has to calculate the asymptotic covariance matrix $\boldsymbol{\Delta}$ of $N^{-1 / 2} \boldsymbol{\Theta}$.

Using (3.1), the sums of squares given in (3.3) can be rewritten in terms of the random vectors $\boldsymbol{\alpha}, \boldsymbol{\beta}, \boldsymbol{e}$, as

$$
\begin{aligned}
& S S A=\left[\boldsymbol{\alpha}^{\prime}, \boldsymbol{\beta}^{\prime}, \boldsymbol{e}^{\prime}\right]\left[\begin{array}{ccc}
\mathbf{Z}_{1}^{\prime} \mathbf{P}_{1} \mathbf{Z}_{1} & \mathbf{Z}_{1}^{\prime} \mathbf{P}_{1} \mathbf{Z}_{2} & \mathbf{Z}_{1}^{\prime} \mathbf{P}_{1} \\
\mathbf{Z}_{2}^{\prime} \mathbf{P}_{1} \mathbf{Z}_{1} & \mathbf{Z}_{2}^{\prime} \mathbf{P}_{1} \mathbf{Z}_{2} & \mathbf{Z}_{2}^{\prime} \mathbf{P}_{1} \\
\mathbf{P}_{1} \mathbf{Z}_{1} & \mathbf{P}_{1} \mathbf{Z}_{2} & \mathbf{P}_{1}
\end{array}\right]\left[\begin{array}{c}
\boldsymbol{\alpha} \\
\boldsymbol{\beta} \\
\boldsymbol{e}
\end{array}\right], \\
& S S B=\left[\boldsymbol{\alpha}^{\prime}, \boldsymbol{\beta}^{\prime}, \boldsymbol{e}^{\prime}\right]\left[\begin{array}{ccc}
\mathbf{0} & \mathbf{0} & \mathbf{0} \\
\mathbf{0} & \mathbf{Z}_{2}^{\prime} \mathbf{P}_{2} \mathbf{Z}_{2} & \mathbf{Z}_{2}^{\prime} \mathbf{P}_{2} \\
\mathbf{0} & \mathbf{P}_{2} \mathbf{Z}_{2} & \mathbf{P}_{2}
\end{array}\right]\left[\begin{array}{c}
\boldsymbol{\alpha} \\
\boldsymbol{\beta} \\
\boldsymbol{e}
\end{array}\right], \\
& S S E=\left[\boldsymbol{\alpha}^{\prime}, \boldsymbol{\beta}^{\prime}, \boldsymbol{e}^{\prime}\right]\left[\begin{array}{ccc}
\mathbf{0} & \mathbf{0} & \mathbf{0} \\
\mathbf{0} & \mathbf{0} & \mathbf{0} \\
\mathbf{0} & \mathbf{0} & \mathbf{P}_{3}
\end{array}\right]\left[\begin{array}{c}
\boldsymbol{\alpha} \\
\boldsymbol{\beta} \\
\boldsymbol{e}
\end{array}\right],
\end{aligned}
$$

where the matrices $\mathbf{Z}_{i}$ and $\mathbf{P}_{i}, i=1,2,3$, are defined in (3.2) and (3.4).

Because $[\boldsymbol{\alpha}, \boldsymbol{\beta}, \boldsymbol{e}]^{\prime}$ is a zero mean random vector, by [15, Lemma 1] one finds that the variances of $S S A, S S B$ and $S S E$ are

$$
\begin{aligned}
\operatorname{Var}(S S A)= & \sigma_{e}^{2}\left(2 \rho_{1}^{2} \operatorname{tr}\left(\mathbf{Z}_{1}^{\prime} \mathbf{P}_{1} \mathbf{Z}_{1}\right)^{2}+4 \rho_{1} \rho_{2} \operatorname{tr}\left(\mathbf{Z}_{1}^{\prime} \mathbf{P}_{1} \mathbf{Z}_{2} \mathbf{Z}_{2}^{\prime} \mathbf{P}_{1} \mathbf{Z}_{1}\right)\right. \\
& +4 \rho_{1} \operatorname{tr}\left(\mathbf{Z}_{1}^{\prime} \mathbf{P}_{1}^{2} \mathbf{Z}_{1}\right)+2 \rho_{2}^{2} \operatorname{tr}\left(\mathbf{Z}_{2}^{\prime} \mathbf{P}_{1} \mathbf{Z}_{2}\right)^{2}+4 \rho_{2} \operatorname{tr}\left(\mathbf{Z}_{2}^{\prime} \mathbf{P}_{1}^{2} \mathbf{Z}_{2}\right) \\
& +2 \operatorname{tr}\left(\mathbf{P}_{1}^{2}\right)+k_{a} \rho_{1}^{2} \operatorname{tr}\left(\mathbf{Z}_{1}^{\prime} \mathbf{P}_{1} \mathbf{Z}_{1} \operatorname{diag}\left(\mathbf{Z}_{1}^{\prime} \mathbf{P}_{1} \mathbf{Z}_{1}\right)\right) \\
& \left.+k_{e} \operatorname{tr}\left(\mathbf{P}_{1} \operatorname{diag}\left(\mathbf{P}_{1}\right)\right)+k_{b} \rho_{2}^{2} \operatorname{tr}\left(\mathbf{Z}_{2}^{\prime} \mathbf{P}_{1} \mathbf{Z}_{2} \operatorname{diag}\left(\mathbf{Z}_{2}^{\prime} \mathbf{P}_{1} \mathbf{Z}_{2}\right)\right)\right), \\
\operatorname{Var}(S S B)= & \sigma_{e}^{2}\left(2 \rho_{2}^{2} \operatorname{tr}\left(\mathbf{Z}_{2}^{\prime} \mathbf{P}_{2} \mathbf{Z}_{2}\right)^{2}+4 \rho_{2} \operatorname{tr}\left(\mathbf{Z}_{2}^{\prime} \mathbf{P}_{2}^{2} \mathbf{Z}_{2}\right)+2 \sigma_{e}^{4} \operatorname{tr}\left(\mathbf{P}_{2}^{2}\right)\right. \\
& \left.+k_{b} \rho_{2}^{2} \operatorname{tr}\left(\mathbf{Z}_{2}^{\prime} \mathbf{P}_{2} \mathbf{Z}_{2} \operatorname{diag}\left(\mathbf{Z}_{2}^{\prime} \mathbf{P}_{2} \mathbf{Z}_{2}\right)\right)+k_{e} \operatorname{tr}\left(\mathbf{P}_{2} \operatorname{diag}\left(\mathbf{P}_{2}\right)\right)\right), \\
\operatorname{Var}(S S E)= & \sigma_{e}^{4}\left(2 \operatorname{tr}\left(\mathbf{P}_{3}^{2}\right)+k_{e} \operatorname{tr}\left(\mathbf{P}_{3} \operatorname{diag}\left(\mathbf{P}_{3}\right)\right)\right)
\end{aligned}
$$

and the covariances (between appropriate sum of squares) are

$$
\begin{aligned}
\operatorname{Cov}(S S A, S S B)= & \sigma_{e}^{4}\left(2 \rho_{1}^{2} \operatorname{tr}\left(\mathbf{Z}_{2}^{\prime} \mathbf{P}_{1} \mathbf{Z}_{2} \mathbf{Z}_{2}^{\prime} \mathbf{P}_{2} \mathbf{Z}_{2}\right)+2 \sigma_{e}^{4} \operatorname{tr}\left(\mathbf{P}_{1} \mathbf{P}_{2}\right)\right. \\
& +4 \rho_{2} \operatorname{tr}\left(\mathbf{Z}_{2}^{\prime} \mathbf{P}_{1} \mathbf{P}_{2} \mathbf{Z}_{2}\right)+k_{b} \rho_{2}^{2} \operatorname{tr}\left(\mathbf{Z}_{2}^{\prime} \mathbf{P}_{1} \mathbf{Z}_{2} \operatorname{diag}\left(\mathbf{Z}_{2}^{\prime} \mathbf{P}_{2} \mathbf{Z}_{2}\right)\right) \\
& \left.+k_{e} \operatorname{tr}\left(\mathbf{P}_{1} \operatorname{diag}\left(\mathbf{P}_{2}\right)\right)\right) \\
\operatorname{Cov}(S S A, S S E)= & \sigma_{e}^{4}\left(2 \operatorname{tr}\left(\mathbf{P}_{1} \mathbf{P}_{3}\right)+k_{e} \operatorname{tr}\left(\mathbf{P}_{1} \operatorname{diag}\left(\mathbf{P}_{3}\right)\right)\right) \\
\operatorname{Cov}(S S B, S S E)= & \sigma_{e}^{4}\left(2 \operatorname{tr}\left(\mathbf{P}_{2} \mathbf{P}_{3}\right)+k_{e} \operatorname{tr}\left(\mathbf{P}_{2} \operatorname{diag}\left(\mathbf{P}_{3}\right)\right)\right)
\end{aligned}
$$


Now, by 3.2 and 3.4 one can easily show that

$$
\begin{aligned}
& \mathbf{Z}_{1}^{\prime} \mathbf{P}_{1} \mathbf{Z}_{1}=\operatorname{diag}\left(n_{1 .}, \ldots, n_{v \cdot}\right)-\frac{1}{N}\left[n_{1}, \ldots, n_{v}\right]^{\prime}\left[n_{1 .}, \ldots, n_{v} .\right] \\
& \mathbf{Z}_{2}^{\prime} \mathbf{P}_{1} \mathbf{Z}_{2}=\bigoplus_{i=1}^{v} \frac{\boldsymbol{n}_{i} \cdot \boldsymbol{n}_{i .}^{\prime}}{n_{i} .}-\frac{1}{N} \boldsymbol{n n}^{\prime}
\end{aligned}
$$

where $\boldsymbol{n}_{i}=\left[n_{i 1}, \ldots, n_{i b_{i}}\right]^{\prime}$ and $\boldsymbol{n}=\left[n_{11}, \ldots, n_{1 b_{1}}, \ldots, n_{v 1}, \ldots, n_{v b_{v}}\right]^{\prime}$.

Hence, by 8.4 ,

$$
\begin{aligned}
& \operatorname{tr}\left(\mathbf{Z}_{1}^{\prime} \mathbf{P}_{1} \mathbf{Z}_{1}\right)^{2}=\sum_{i=1}^{v} n_{i .}^{2}-2 \frac{1}{N} \sum_{i=1}^{v} n_{i .}^{3}+\frac{1}{N^{2}} \sum_{i=1}^{v} \sum_{l=1}^{v} n_{i}^{2} n_{l .}^{2}, \\
& \operatorname{tr}\left(\mathbf{Z}_{1}^{\prime} \mathbf{P}_{1} \mathbf{Z}_{1} \operatorname{diag}\left(\mathbf{Z}_{1}^{\prime} \mathbf{P}_{1} \mathbf{Z}_{1}\right)\right)=\sum_{i=1}^{v} n_{i}^{2}\left(1-\frac{1}{N}\right)^{2},
\end{aligned}
$$

Since the matrix $\mathbf{P}_{1}$ is idempotent, one has

$$
\operatorname{tr}\left(\mathbf{Z}_{1}^{\prime} \mathbf{P}_{1}^{2} \mathbf{Z}_{1}\right)=\operatorname{tr}\left(\mathbf{Z}_{1}^{\prime} \mathbf{P}_{1} \mathbf{Z}_{1}\right)=\sum_{i=1}^{v}\left(n_{i}-\frac{n_{i}}{N}\right)
$$

Thus, by Lemma 3.3 .

$$
\begin{aligned}
\lim _{N \rightarrow \infty} \frac{1}{N} \operatorname{tr}\left(\mathbf{Z}_{1}^{\prime} \mathbf{P}_{1} \mathbf{Z}_{1}\right)^{2} & =\gamma_{2}=\lim _{N \rightarrow \infty} \frac{1}{N} \operatorname{tr}\left(\mathbf{Z}_{1}^{\prime} \mathbf{P}_{1} \mathbf{Z}_{1} \operatorname{diag}\left(\mathbf{Z}_{1}^{\prime} \mathbf{P}_{1} \mathbf{Z}_{1}\right)\right), \\
\lim _{N \rightarrow \infty} \frac{1}{N} \operatorname{tr}\left(\mathbf{Z}_{1}^{\prime} \mathbf{P}_{1}^{2} \mathbf{Z}_{1}\right) & =\lim _{N \rightarrow \infty} \frac{1}{N} \operatorname{tr}\left(\mathbf{Z}_{1}^{\prime} \mathbf{P}_{1} \mathbf{Z}_{1}\right)=1
\end{aligned}
$$

Using similar arguments, one can show that

$$
\begin{aligned}
\operatorname{tr}\left(\mathbf{Z}_{2}^{\prime} \mathbf{P}_{1} \mathbf{Z}_{2}\right)^{2} & =\sum_{i=1}^{v} \frac{\left(\sum_{j=1}^{b_{i}} n_{i j}^{2}\right)^{2}}{n_{i}^{2}}+\frac{1}{N^{2}}\left(\sum_{i=1}^{v} \sum_{j=1}^{b_{i}} n_{i j}^{2}\right)^{2}-\frac{2}{N} \boldsymbol{n}^{\prime}\left(\bigoplus_{i=1}^{v} \frac{\boldsymbol{n}_{i} \boldsymbol{n}_{i}^{\prime}}{n_{i} .}\right) \boldsymbol{n} \\
& =\sum_{i=1}^{v} \frac{\left(\sum_{j=1}^{b_{i}} n_{i j}^{2}\right)^{2}}{n_{i}^{2}}+\frac{1}{N^{2}}\left(\sum_{i=1}^{v} \sum_{j=1}^{b_{i}} n_{i j}^{2}\right)^{2}-\frac{2}{N} \sum_{i=1}^{v} \frac{\left(\sum_{i=1}^{b_{i}} n_{i j}^{2}\right)^{2}}{n .} .
\end{aligned}
$$

Moreover,

$$
\begin{aligned}
& \operatorname{tr}\left(\mathbf{Z}_{2}^{\prime} \mathbf{P}_{1} \mathbf{Z}_{2} \operatorname{diag}\left(\mathbf{Z}_{2}^{\prime} \mathbf{P}_{1} \mathbf{Z}_{2}\right)\right)=\sum_{i=1}^{v} \sum_{j=1}^{b_{i}} n_{i j}^{4}\left(\frac{1}{n_{i}}-\frac{1}{N}\right)^{2}, \\
& \operatorname{tr}\left(\mathbf{Z}_{2}^{\prime} \mathbf{P}_{1}^{2} \mathbf{Z}_{2}\right)=\operatorname{tr}\left(\mathbf{Z}_{2}^{\prime} \mathbf{P}_{1} \mathbf{Z}_{2}\right)=\sum_{i=1}^{v} \sum_{j=1}^{b_{i}} n_{i j}^{2}\left(\frac{1}{n_{i}}-\frac{1}{N}\right) .
\end{aligned}
$$


Thus, by Lemmas 3.3 and 3.4 .

$$
\begin{aligned}
& \lim _{N \rightarrow \infty} \frac{1}{N} \operatorname{tr}\left(\mathbf{Z}_{2}^{\prime} \mathbf{P}_{1} \mathbf{Z}_{2}\right)^{2}=\vartheta, \\
& \lim _{N \rightarrow \infty} \frac{1}{N} \operatorname{tr}\left(\mathbf{Z}_{2}^{\prime} \mathbf{P}_{1} \mathbf{Z}_{2} \operatorname{diag}\left(\mathbf{Z}_{2}^{\prime} \mathbf{P}_{1} \mathbf{Z}_{2}\right)\right)=\delta_{24}, \\
& \lim _{N \rightarrow \infty} \operatorname{tr}\left(\mathbf{Z}_{2}^{\prime} \mathbf{P}_{1}^{2} \mathbf{Z}_{2}\right)=\lim _{N \rightarrow \infty} \frac{1}{N} \operatorname{tr}\left(\mathbf{Z}_{2}^{\prime} \mathbf{P}_{1} \mathbf{Z}_{2}\right)=\delta_{12} .
\end{aligned}
$$

Because the matrix $\mathbf{P}_{1}$ is idempotent, one has

$$
\operatorname{tr} \mathbf{P}_{1}^{2}=\operatorname{tr} \mathbf{P}_{1}=\sum_{i=1}^{v} n_{i} \cdot\left(\frac{1}{n_{i}}-\frac{1}{N}\right)=v-1 .
$$

Also, one can easily show that

$$
\operatorname{tr}\left(\mathbf{P}_{1} \operatorname{diag}\left(\mathbf{P}_{1}\right)\right)=\sum_{i=1}^{v} n_{i} \cdot\left(\frac{1}{n_{i}}-\frac{1}{N}\right)^{2} .
$$

Hence,

$$
\lim _{N \rightarrow \infty} \frac{1}{N} \operatorname{tr} \mathbf{P}_{1}^{2}=\zeta, \quad \lim _{N \rightarrow \infty} \frac{1}{N} \operatorname{tr}\left(\mathbf{P}_{1} \operatorname{diag}\left(\mathbf{P}_{1}\right)\right)=\gamma_{-1} .
$$

After some algebra using the properties of matrix trace (see [11]) it follows that

$$
\begin{aligned}
\operatorname{tr}\left(\mathbf{Z}_{1}^{\prime} \mathbf{P}_{1} \mathbf{Z}_{2} \mathbf{Z}_{2}^{\prime} \mathbf{P}_{1} \mathbf{Z}_{1}\right) & =\operatorname{tr}\left(\left(\bigoplus_{i=1}^{v} \bigoplus_{j=1}^{b_{i}} \mathbf{J}_{n_{i j}}\right) \mathbf{P}_{1}\left(\bigoplus_{i=1}^{v} \mathbf{J}_{n_{i} .}\right) \mathbf{P}_{1}\right) \\
& =\sum_{i=1}^{v} \sum_{j=1}^{b_{i}} n_{i j}^{2}-\frac{1}{N} \sum_{i=1}^{v} n_{i}^{2},
\end{aligned}
$$

and by Lemma 3.4 ,

$$
\lim _{N \rightarrow \infty} \frac{1}{N} \operatorname{tr}\left(\mathbf{Z}_{1}^{\prime} \mathbf{P}_{1} \mathbf{Z}_{2} \mathbf{Z}_{2}^{\prime} \mathbf{P}_{1} \mathbf{Z}_{1}\right)=\delta_{02} .
$$

Thus by 8.6 8.9 ,

$$
\begin{array}{r}
\lim _{N \rightarrow \infty} \frac{1}{N} \operatorname{Var}(S S A)=\sigma_{e}^{2}\left(2 \rho_{1}^{2} \gamma_{2}+4 \rho_{1} \rho_{2} \delta_{02}+4 \rho_{1}+4 \rho_{2} \delta_{12}+2 \sigma_{b}^{4} \vartheta\right. \\
\left.+2 \sigma_{e}^{4}+k_{a} \sigma_{a}^{4} \gamma_{2}+k_{b} \sigma_{b}^{4} \delta_{24}+k_{e} \sigma_{e}^{4} \gamma_{-1}\right) .
\end{array}
$$

Using (3.2) and (3.4), one writes

$$
\mathbf{Z}_{2}^{\prime} \mathbf{P}_{2} \mathbf{Z}_{2}=\operatorname{diag}\left(n_{11}, \ldots, n_{1 b 1}, \ldots, n_{v 1}, \ldots, n_{v b_{v}}\right)-\bigoplus_{i=1}^{v} \frac{\boldsymbol{n}_{i} . \boldsymbol{n}_{i}^{\prime}}{n_{i}} .
$$


Hence,

$$
\operatorname{tr}\left(\mathbf{Z}_{2}^{\prime} \mathbf{P}_{2} \mathbf{Z}_{2} \operatorname{diag}\left(\mathbf{Z}_{2}^{\prime} \mathbf{P}_{2} \mathbf{Z}_{2}\right)\right)=\sum_{i=1}^{v} \sum_{j=1}^{b_{i}} n_{i j}^{2}\left(1-\frac{n_{i j}}{n_{i}}\right)^{2},
$$

and after some algebra

$$
\operatorname{tr}\left(\mathbf{Z}_{2}^{\prime} \mathbf{P}_{2} \mathbf{Z}_{2}\right)^{2}=\sum_{i=1}^{v} \sum_{j=1}^{b_{i}} n_{i j}^{2}-2 \sum_{i=1}^{v} \sum_{j=1}^{b_{i}} \frac{n_{i j}^{3}}{n_{i} .}+\sum_{i=1}^{v} \frac{\left(\sum_{j=1}^{b_{i}} n_{i j}^{2}\right)^{2}}{n_{i}^{2} .} .
$$

Hence, by Lemma 3.4 .

$$
\begin{aligned}
& \lim _{N \rightarrow \infty} \frac{1}{N} \operatorname{tr}\left(\mathbf{Z}_{2}^{\prime} \mathbf{P}_{2} \mathbf{Z}_{2} \operatorname{diag}\left(\mathbf{Z}_{2}^{\prime} \mathbf{P}_{2} \mathbf{Z}_{2}\right)\right)=\delta_{02}-2 \delta_{13}+\delta_{24}, \\
& \lim _{N \rightarrow \infty} \frac{1}{N} \operatorname{tr}\left(\mathbf{Z}_{2}^{\prime} \mathbf{P}_{2} \mathbf{Z}_{2}\right)^{2}=\delta_{02}-2 \delta_{13}+\vartheta .
\end{aligned}
$$

The matrix $\mathbf{P}_{2}$ is idempotent, so $\operatorname{tr} \mathbf{P}_{2}^{2}=\operatorname{tr} \mathbf{P}_{2}$, where $\operatorname{tr} \mathbf{P}_{2}=b$. $-v$, and

$$
\operatorname{tr}\left(\mathbf{Z}_{2}^{\prime} \mathbf{P}_{2}^{2} \mathbf{Z}_{2}\right)=\operatorname{tr}\left(\mathbf{Z}_{2}^{\prime} \mathbf{P}_{2} \mathbf{Z}_{2}\right)=\sum_{i=1}^{v} \sum_{j=1}^{b_{i}}\left(n_{i j}-\frac{n_{i j}^{2}}{n_{i} .}\right) .
$$

Moreover,

$$
\operatorname{tr}\left(\mathbf{P}_{2} \operatorname{diag}\left(\mathbf{P}_{2}\right)\right)=\sum_{i=1}^{v} \sum_{j=1}^{b_{i}} n_{i j}\left(\frac{1}{n_{i j}}-\frac{1}{n_{i}}\right) .
$$

This implies that

$$
\begin{aligned}
& \lim _{N \rightarrow \infty} \frac{1}{N} \operatorname{tr}\left(\mathbf{P}_{2}^{2}\right)=\varphi-\zeta, \\
& \lim _{N \rightarrow \infty} \frac{1}{N} \operatorname{tr}\left(\mathbf{P}_{2} \operatorname{diag}\left(\mathbf{P}_{2}\right)\right)=\eta-2 \delta+\gamma_{-1}, \\
& \lim _{N \rightarrow \infty} \frac{1}{N} \operatorname{tr}\left(\mathbf{Z}_{2}^{\prime} \mathbf{P}_{2}^{2} \mathbf{Z}_{2}\right)=\lim _{N \rightarrow \infty} \frac{1}{N} \operatorname{tr}\left(\mathbf{Z}_{2}^{\prime} \mathbf{P}_{2} \mathbf{Z}_{2}\right)=1-\delta_{12} .
\end{aligned}
$$

Combining 8.12 and 8.13 one gets

$$
\begin{aligned}
\lim _{N \rightarrow \infty} \frac{1}{N} \operatorname{Var}(S S B)=\sigma_{e}^{4}\left(2 \rho_{2}^{2}\left(\delta_{02}-2 \delta_{13}+\vartheta\right)+4 \rho_{2}\left(1-\delta_{12}\right)\right. \\
\left.+2(\varphi-\zeta)+k_{b} \rho_{2}^{2}\left(\delta_{02}-2 \delta_{13}+\delta_{24}\right)+k_{e}\left(\eta-2 \delta+\gamma_{-1}\right)\right) .
\end{aligned}
$$

The matrix $\mathbf{P}_{3}$ is idempotent, so

$$
\operatorname{tr}\left(\mathbf{P}_{3}^{2}\right)=\operatorname{tr}\left(\mathbf{P}_{3}\right)=\sum_{i=1}^{v} \sum_{j=1}^{b_{i}} n_{i j}\left(1-\frac{1}{n_{i}}\right)=N-b .
$$


and furthermore

$$
\operatorname{tr}\left(\mathbf{P}_{3} \operatorname{diag}\left(\mathbf{P}_{3}\right)\right)=\sum_{i=1}^{v} \sum_{j=1}^{b_{i}} n_{i j}\left(1-\frac{1}{n_{i j}}\right)^{2}=N-2 b .+\sum_{i=1}^{v} \sum_{j=1}^{b_{i}} \frac{1}{n_{i j}} .
$$

This implies that

$$
\lim _{N \rightarrow \infty} \frac{1}{N} \operatorname{tr}\left(\mathbf{P}_{3}^{2}\right)=1-\varphi, \quad \lim _{N \rightarrow \infty} \frac{1}{N} \operatorname{tr}\left(\mathbf{P}_{3} \operatorname{diag}\left(\mathbf{P}_{3}\right)\right)=1-2 \varphi+\eta .
$$

Hence,

$$
\lim _{N \rightarrow \infty} \operatorname{Var}(S S E)=2 \sigma_{e}^{4}(1-\varphi)+k_{e} \sigma_{e}^{4}(1-2 \varphi+\eta) .
$$

Now, by the definition of $\mathbf{P}_{i}, i=1,2,3$,

$$
\begin{aligned}
& \operatorname{tr}\left(\mathbf{P}_{1} \operatorname{diag}\left(\mathbf{P}_{2}\right)\right)=\sum_{i=1}^{v} \sum_{j=1}^{b_{i}} n_{i j}\left(\frac{1}{n_{i}}-\frac{1}{N}\right)\left(\frac{1}{n_{i j}}-\frac{1}{n_{i} .}\right), \\
& \operatorname{tr}\left(\mathbf{P}_{1} \operatorname{diag}\left(\mathbf{P}_{3}\right)\right)=\sum_{i=1}^{v} \sum_{j=1}^{b_{i}} n_{i j}\left(\frac{1}{n_{i}}-\frac{1}{N}\right)\left(1-\frac{1}{n_{i j}}\right), \\
& \operatorname{tr}\left(\mathbf{P}_{2} \operatorname{diag}\left(\mathbf{P}_{3}\right)\right)=\sum_{i=1}^{v} \sum_{j=1}^{b_{i}} n_{i j}\left(\frac{1}{n_{i j}}-\frac{1}{n_{i}}\right)\left(1-\frac{1}{n_{i j}}\right) .
\end{aligned}
$$

This implies

$$
\begin{aligned}
\lim _{N \rightarrow \infty} \frac{1}{N} \operatorname{tr}\left(\mathbf{P}_{1} \operatorname{diag}\left(\mathbf{P}_{2}\right)\right) & =\delta-\gamma_{-1}, \\
\lim _{N \rightarrow \infty} \frac{1}{N} \operatorname{tr}\left(\mathbf{P}_{1} \operatorname{diag}\left(\mathbf{P}_{3}\right)\right) & =\zeta-\delta, \\
\lim _{N \rightarrow \infty} \frac{1}{N} \operatorname{tr}\left(\mathbf{P}_{2} \operatorname{diag}\left(\mathbf{P}_{3}\right)\right) & =\varphi-\eta-\zeta+\delta .
\end{aligned}
$$

By (8.5) and (8.11) after some algebra one obtains

$$
\begin{aligned}
\operatorname{tr}\left(\mathbf{Z}_{2}^{\prime} \mathbf{P}_{1} \mathbf{Z}_{2} \mathbf{Z}_{2}^{\prime} \mathbf{P}_{2} \mathbf{Z}_{2}\right)= & \sum_{i=1}^{v} \sum_{j=1}^{b_{i}} \frac{n_{i j}^{3}}{n_{i}}-\sum_{i=1}^{v} \frac{\left(\sum_{j=1}^{b_{i}} n_{i j}^{2}\right)^{2}}{n_{i}^{2}} \\
& -\frac{1}{N} \sum_{i=1}^{v} \sum_{j=1}^{b_{i}} n_{i j}^{3}+\frac{1}{N} \sum_{i=1}^{v} \frac{\left(\sum_{j=1}^{b_{i}} n_{i j}^{2}\right)^{2}}{n_{i} .} \\
\operatorname{tr}\left(\mathbf{Z}_{2}^{\prime} \mathbf{P}_{1} \mathbf{Z}_{2} \operatorname{diag}\left(\mathbf{Z}_{2}^{\prime} \mathbf{P}_{2} \mathbf{Z}_{2}\right)\right)= & \sum_{i=1}^{v} \sum_{j=1}^{b_{i}} n_{i j}^{3}\left(1-\frac{n_{i j}}{n_{i}}\right)\left(\frac{1}{n_{i} .}-\frac{1}{N}\right) .
\end{aligned}
$$

Because the matrices $\mathbf{P}_{i}, i=1,2,3$, are pairwise orthogonal, i.e. $\mathbf{P}_{i} \mathbf{P}_{j}=\mathbf{0}$ 
for $i \neq j$, by 8.17 and 8.18 one obtains

$$
\begin{aligned}
& \lim _{N \rightarrow \infty} \frac{1}{N} \operatorname{Cov}(S S A, S S B) \\
& =\sigma_{e}^{4}\left(2 \rho_{2}^{2}\left(\delta_{13}-\vartheta\right)+k_{b} \rho_{2}^{2}\left(\delta_{13}-\delta_{24}\right)+k_{e}\left(\delta-\gamma_{1}\right)\right), \\
& \lim _{N \rightarrow \infty} \frac{1}{N} \operatorname{Cov}(S S A, S S E)=k_{e} \sigma_{e}^{4}(\zeta-\delta), \\
& \lim _{N \rightarrow \infty} \frac{1}{N} \operatorname{Cov}(S S B, S S E)=k_{e} \sigma_{e}^{4}(\varphi-\eta-\zeta+\delta) .
\end{aligned}
$$

Thus, by (8.10, (8.14), 8.16) and 8.19 the asymptotic covariance matrix of $N^{-1 / 2} \boldsymbol{\Theta}$ is given by

$$
\boldsymbol{\Delta}=\sigma_{e}^{4}\left(\boldsymbol{\Xi}_{1}+\boldsymbol{\Xi}_{2}+\boldsymbol{\Xi}_{3}+\boldsymbol{\Xi}_{4}\right),
$$

where

$$
\begin{aligned}
& \boldsymbol{\Xi}_{1}= \\
& \left.\begin{array}{lll}
2\left(\rho_{1}^{2} \gamma_{2}+2 \rho_{1} \rho_{2} \delta_{02}+2 \rho_{1}+2 \rho_{2} \delta_{12}+\rho_{2}^{2} \vartheta+\zeta\right) & 2 \rho_{2}^{2}\left(\delta_{13}-\vartheta\right) & 0 \\
2 \rho_{2}^{2}\left(\delta_{13}-\vartheta\right) & 2\left(\rho_{2}^{2}\left(\delta_{02}-2 \delta_{13}+\vartheta\right)+2 \rho_{2}\left(1-\delta_{12}\right)+\varphi-\zeta\right) & 0 \\
0 & 0
\end{array}\right], \\
& \boldsymbol{\Xi}_{2}=\left[\begin{array}{lll}
\gamma_{2} & 0 & 0 \\
0 & 0 & 0 \\
0 & 0 & 0
\end{array}\right], \quad \boldsymbol{\Xi}_{3}=\left[\begin{array}{lll}
\delta_{24} & \delta_{13}-\delta_{24} & 0 \\
\delta_{13}-\delta_{24} & \delta_{02}-2 \delta_{13}+\delta_{24} & 0 \\
0 & 0 & 0
\end{array}\right],
\end{aligned}
$$

and

$$
\boldsymbol{\Xi}_{4}=\left[\begin{array}{lll}
\gamma_{-1} & \delta-\gamma_{-1} & \varphi-\delta \\
\delta-\gamma_{-1} & \eta+\gamma_{-1}-2 \delta & \varphi-\eta-\zeta+\delta \\
\varphi-\delta & \varphi-\eta-\zeta+\delta & 1-2 \varphi+\eta
\end{array}\right]
$$

This completes the proof of Theorem 4.1.

Proof of Proposition 4.2. First, we will show that

$$
\sqrt{N}(\boldsymbol{\theta}-E(\boldsymbol{\theta})) \stackrel{d}{\rightarrow} N(\mathbf{0}, \boldsymbol{\Sigma}) \quad \text { as } N \rightarrow \infty .
$$

In view of the Main Theorem in [14, in order to prove (8.21) it suffices to show that the assumptions of that theorem are satisfied. Note that assumptions (A2)-(A4) are equivalent to (i)-(iii) in [14]. Thus, one only has to show that assumptions (iv) and (v) are satisfied.

By the proof of Theorem 4.1 (see 8.20), under (A1)-(A4),

$$
\frac{1}{N} \operatorname{Var}(\boldsymbol{\Theta}) \rightarrow \boldsymbol{\Delta} \quad \text { as } N \rightarrow \infty .
$$

Hence, condition (v) of the Main Theorem is satisfied.

Furthermore, by (A2)-(A4), $\boldsymbol{\Omega}_{N} \rightarrow \boldsymbol{\Omega}$ as $N \rightarrow \infty$ (see the proof o Theorem 4.1. Thus, taking $F_{N}=\boldsymbol{\Omega}_{N}^{-1}$ and $F=\boldsymbol{\Omega}^{-1}$ in assumption (iv) in [14, 
one obtains

$$
\sqrt{N}(\boldsymbol{\theta}-E(\boldsymbol{\theta})) \stackrel{d}{\rightarrow} N\left(\mathbf{0}, \boldsymbol{\Omega} \boldsymbol{\Delta} \boldsymbol{\Omega}^{\prime}\right) \quad \text { as } N \rightarrow \infty .
$$

By Theorem 4.1 one gets the claim.

Now, after some algebra using the identity $E\left(\boldsymbol{a}^{\prime} \mathbf{A} \boldsymbol{a}\right)=\operatorname{tr}(\mathbf{A} \boldsymbol{\Lambda})+\boldsymbol{\mu}^{\prime} \mathbf{A} \boldsymbol{\mu}$, we have

$$
E\left(\boldsymbol{\theta}_{N}\right)=\sigma_{e}^{2}\left[\begin{array}{l}
\frac{1}{v-1}\left(\rho_{1} \operatorname{tr}\left(\mathbf{Z}_{1}^{\prime} \mathbf{P}_{1} \mathbf{Z}_{1}\right)+\rho_{2} \operatorname{tr}\left(\mathbf{Z}_{2}^{\prime} \mathbf{P}_{1} \mathbf{Z}_{2}\right)+\operatorname{tr}\left(\mathbf{P}_{1}\right)\right) \\
\frac{1}{b .-v}\left(\rho_{2} \operatorname{tr}\left(\mathbf{Z}_{2}^{\prime} \mathbf{P}_{2} \mathbf{Z}_{2}\right)+\operatorname{tr}\left(\mathbf{P}_{2}\right)\right) \\
\frac{1}{N-b .} \operatorname{tr}\left(\mathbf{P}_{3}\right)
\end{array}\right] .
$$

Then, by $8.6-8.9), 8.13$ and 8.15 it follows that

$$
E\left(\boldsymbol{\theta}_{N}\right) \rightarrow \boldsymbol{\Gamma} \text { as } N \rightarrow \infty .
$$

Combining 8.21 and 8.22 we get the assertion.

Proof of Theorem 4.3. (i) Note that $F_{1}=M S B / M S E$ is a function of $M S A, M S B$ and $M S E$. Thus, differentiating $F_{1}$ with respect to $M S A, M S B$ and $M S E$ at

$$
\left(\sigma_{e}^{2}\left(\rho_{1} \zeta^{-1}+\rho_{2} \delta_{12} \zeta^{-1}+1\right), \sigma_{e}^{2}\left(1+\rho_{2}\left(1-\delta_{12}\right)(\varphi-\zeta)^{-1}\right), \sigma_{e}^{2}\right),
$$

one finds that the vector $\nabla F_{1}$ of first derivatives is equal to

$$
\nabla F_{1}=\left[0, \frac{1}{\sigma_{e}^{2}},-\frac{1+\rho_{2}\left(1-\delta_{12}\right)(\varphi-\zeta)^{-1}}{\sigma_{e}^{2}}\right]^{\prime}
$$

Hence, by using the delta method (see e.g. [12]) one has

$$
\sqrt{N}\left(F_{1}-\left(1+\frac{\rho_{2}\left(1-\delta_{12}\right)}{\varphi-\zeta}\right)\right)=\sqrt{N}\left(\nabla F_{1}\right)^{\prime}(\boldsymbol{\theta}-\boldsymbol{\Gamma})+o_{P}(1) .
$$

Thus, by [3, Theorem 4.2 and Proposition 5.2],

$$
\sqrt{N}\left(F_{1}-\left(1+\frac{\rho_{2}\left(1-\delta_{12}\right)}{\varphi-\zeta}\right)\right) \stackrel{d}{\rightarrow} N\left(0, \sigma_{H_{1}}^{2}\right),
$$

where $\sigma_{H_{1}}^{2}=\left(\nabla F_{1}\right)^{\prime} \boldsymbol{\Sigma}\left(\nabla F_{1}\right)$. After some algebra one gets (i).

(ii) Using similar arguments to those in (i) one finds that

$$
\sqrt{N}\left(F_{2}-\widetilde{F}\right)=\sqrt{N}\left(\nabla F_{2}\right)^{\prime}(\boldsymbol{\theta}-\boldsymbol{\Gamma})+o_{P}(1),
$$

where $\widetilde{F}$ and $\nabla F_{2}$ are the value of $F_{2}$ and the vector of first derivatives of $F_{2}$ calculated at 8.23 , and are equal to

$$
\begin{aligned}
\widetilde{F} & =1+\frac{\rho_{1} \zeta^{-1}+\rho_{2} \delta_{12} \zeta^{-1}-\rho_{2}\left(1-\delta_{12}\right)(\varphi-\zeta)^{-1}}{1+\rho_{2}\left(1-\delta_{12}\right)(\varphi-\zeta)^{-1}}, \\
\nabla F_{2} & =\left[\frac{1}{\sigma_{e}^{2}\left(1+\rho_{2}\left(1-\delta_{12}\right)(\varphi-\zeta)^{-1}\right)}, \frac{-\left(\rho_{1} \zeta^{-1}+\rho_{2} \delta_{12} \zeta^{-1}+1\right)}{\sigma_{e}^{2}\left(1+\rho_{2}\left(1-\delta_{12}\right)(\varphi-\zeta)^{-1}\right)^{2}}, 0\right]^{\prime} .
\end{aligned}
$$


Hence, by Theorem $4.2 \sqrt{N}\left(F_{2}-\widetilde{F}\right)$ is asymptotically normal with variance $\sigma_{H_{2}}^{2}=\left(\nabla F_{2}\right)^{\prime} \boldsymbol{\Sigma}\left(\nabla F_{2}\right)$. After straightforward computations one gets (ii).

\section{References}

[1] M. Akritas and S. Arnold, Asymptotics for analysis of variance when the number of levels is large, J. Amer. Statist. Assoc. 95 (2000), 212-226.

[2] A. Azzalini and A. Capitanio, Statistical applications of the multivariate skewnormal distribution, J. Roy. Statist. Soc. Ser. B 61 (1999), 579-602.

[3] M. Bilodeau and D. Brenner, Theory of Multivariate Statistics, Springer, New York, 1999.

[4] W. B. Cummings and D. W. Gaylor, Variance component testing in unbalanced nested designs, J. Amer. Statist. Assoc. 69 (1974), 765-771.

[5] R. Elandt, Mathematical Statistics with Application to Agricultural Experimentation, PWN, Warszawa, 1964 (in Polish).

[6] G. M. Fitzmaurice, S. R. Lipsitz and J. G. Ibrahim, A note on permutation test for variance components in multilevel generalized linear mixed models, Biometrics 63 (2007), 942-946.

[7] B. Güven, The limiting distribution of the F-statistic from nonnormal universes, Statistics 40 (2006), 545-557.

[8] B. Güven, Approximate tests in unbalanced two-way random models without interaction, Statist. Papers 53 (2012), 753-766.

[9] J. Jiang, Linear and Generalized Linear Mixed Models and Their Applications, Springer, New York, 2007.

[10] A. I. Khuri, T. Mathew and B. K Sinha, Statistical Tests for Mixed Linear Models, Wiley, New York, 1998.

[11] J. R. Magnus and H. Neudecker, Matrix Differential Calculus with Applications in Statistics and Econometrics, Wiley, New York, 1999.

[12] A. van der Vaart, Asymptotic Statistics, Cambridge Univ. Press, Cambridge, 1998.

[13] J. Volaufová, Heteroscedastic ANOVA: old p-values, new views, Statist. Papers 50 (2009), 943-962.

[14] P. H. Westfall, Asymptotic normality of the ANOVA estimates of components of variance in the nonnormal, unbalanced hierarchal mixed model, Ann. Statist. 14 (1986), 1572-1582.

[15] P. H. Westfall, A comparison of variance component estimates for arbitrary underlying distributions, J. Amer. Statist. Assoc. 82 (1987), 866-874.

[16] B. J. Winer, Statistical Principles in Experimental Design, McGraw-Hill, New York, 1962.

Marcin Przystalski

The Research Centre for Cultivar Testing

63-022 Słupia Wielka, Poland

E-mail: marprzyst@gmail.com 